Article

\title{
Occurrence, Antimicrobial Resistance and Molecular Diversity of Enterococcus faecium in Processed Pork Meat Products in Korea
}

\author{
Hyun Jung Kim ${ }^{1,2, *(D)}$ and Minseon Koo ${ }^{2,3}$ \\ 1 Research Group of Consumer Safety, Korea Food Research Institute, Wanju, Jeollabuk-do 55365, Korea \\ 2 Department of Food Biotechnology, University of Science and Technology, Daejeon 34113, Korea; \\ minsk@kfri.re.kr \\ 3 Food Analysis Center, Korea Food Research Institute, Wanju, Jeollabuk-do 55365, Korea \\ * Correspondence: hjkim@kfri.re.kr; Tel.: +82-63-219-9271
}

Received: 9 July 2020; Accepted: 9 September 2020; Published: 12 September 2020

\begin{abstract}
Because Enterococcus faecium is an important nosocomial pathogen and sentinel organism for tracking antimicrobial resistance, information on the contamination and antimicrobial resistance patterns of E. faecium in food are essential to public health and food safety. We analyzed the occurrence of E. faecium in retail pork meat products $(n=124)$, and antimicrobial resistance of 30 E. faecium isolates were examined against 14 antimicrobials using the broth dilution test and disc diffusion test. Rep-PCR-based molecular diversity was also analyzed using Deviersilab. The highest contamination of enterococci was observed for minced pork meat but most of the E. faecium was isolated from meatball-type frozen pork meat products (FP). Incidences of antimicrobial-resistant E. faecium against erythromycin, clindamycin and nitrofurantoin were $80 \%, 50 \%$ and $20 \%$, respectively. No vancomycin-resistant enterococci were analyzed. Rep-PCR showed distinctive clusters with a similarity $\geq 98 \%$, consisting of 18 E. faecium isolates from FP manufactured in seven companies. The analyzed data on the contamination and antimicrobial resistance patterns combined with molecular typing can be useful to derive risk management of antimicrobial-resistant enterococci in food.
\end{abstract}

Keywords: Enterococcus faecium; processed pork meat products; antimicrobial resistance; rep-PCR; molecular typing

\section{Introduction}

Enterococci are gram-positive, facultative anaerobic bacteria that are frequently detected in the intestinal tracts of animals and humans as a part of the normal microbiota [1]. Generally they are not harmful to humans, but some species of Enterococcus are known as nosocomial agents in hospitalized and immunocompromised patients in hematology, oncology, and transplantation surgery [1]. Enterococci are a major cause of sepsis worldwide and are among the leading nosocomial pathogens and account for about $10 \%$ of hospital-acquired bacteremia cases [2]. Antimicrobial resistance is known to contribute to increased morbidity and mortality in infections caused by Enterococcus [3]. They can be transmitted not only person-to-person, but also through contaminated environments including foods, causing a variety of infections, such as bacteremia, endocarditis, and urinary tract infections [4-7].

As enterococci are part of the normal microbiota of animals and they are able to survive in biotic and abiotic environments, they are also found in foods of animal and plant origin. Enterococci are recognized as a hygienic indicator in foods and as sentinel organisms for tracking antimicrobial resistance trends $[1,8]$. In the food industry, some strains of enterococci have been used as probiotics for human and animal use, as well as starter cultures for fermented food production [5,9]. Enterococci 
play a beneficial role in the production of traditional fermented foods, giving unique organoleptic properties $[5,10]$. However, their ability to harbor and easily acquire virulence and antibiotic resistance genes through horizontal gene transfer hampers their use as probiotics or as starter/adjunct cultures in foods [11].

The most frequently detected enterococcus species in clinical and food samples of greatest importance to human health are Enterococcus faecalis and Enterococcus faecium. Both species cause a variety of infections in immunocompromised patients [7]. They show resistance to antimicrobials including $\beta$-lactams, high-levels of aminoglycosides, and glycopeptides [6]. Formerly, 90\% of the human infections caused by enterococci were due to E. faecalis, and the remaining $10 \%$ were known to be related to E. faecium [11,12]. However, recent data have shown that much more frequent incidences of antimicrobial resistance against vancomycin, ampicillin, and high levels of aminoglycosides can be observed in E. faecium compared to E. faecalis [11,13]. Antimicrobial-resistant E. faecium as a conditional pathogen with low pathogenicity affects above all immunocompromised patients and possibly cause systemic infections limiting the choice of an effective antibiotics.

Foodborne transmission of enterococci may affect a larger part of the population through consumption and handling of contaminated poultry meat and other food items that may be cross-contaminated in the kitchen [14]. Despite the availability of anti-gram-positive agents (e.g., linezolid, quinupristin/dalfopristin, daptomycin, tigecycline), enterococci have rapidly adapted and resistance has emerged to all these newer agents [15]. Because foods are important vehicles for transmitting antimicrobial-resistant enterococci, contamination and antimicrobial resistance patterns of enterococci in food are essential to public health and food safety with respect to horizontal transfer of genes conferring resistance to antibiotics that are relevant for treatment of enterococcal infections.

However, most studies on the prevalence of antimicrobial-resistant enterococci have focused on E. faecalis in Korea. Processed pork meat products are popular food dishes that are frequently provided in restaurants and school canteen services in Korea [16], possible contamination of antimicrobial-resistant E. faecium in pork meat dishes can cause a food safety and public health burden. However, information on the antimicrobial resistance of E. faecium, especially in retail pork meat products, is limited [17]. In this study, the occurrence of E. faecium in retail pork meat products and the antimicrobial resistance of the isolates were examined. Automated repetitive-sequence-based PCR (rep-PCR) was used to investigate the strain relatedness of E. faecium isolates obtained from pork meat products and to provide molecular epidemiology data useful for risk management of enterococci with particular antibiotic resistance traits.

\section{Materials and Methods}

\subsection{Sample Collection and Preparation}

Samples of processed pork meat products were collected from eight retail markets located in Gyonggi Province in South Korea. The 124 samples included minced pork meat (MP, $n=40)$, ready-to-cook pork meat products seasoned with fermented red pepper paste $(S R P, n=34)$ or seasoned with soy sauce (SSP, $n=34$ ), and meatball-type frozen processed pork meat products (FP, $n=16$ ). Samples were placed on ice in a cooling box after purchase and were transported to the laboratory within $2 \mathrm{~h}$. Samples that included MP, SRP and SSP were immediately stored at $4{ }^{\circ} \mathrm{C}$. FP samples were kept at $-20^{\circ} \mathrm{C}$ until analysis.

\subsection{Isolation and Identification of Enterococcus spp.}

The enumeration of enterococci was carried out according to ISO-7899-1 [9,18] with some modifications. In brief, $25 \mathrm{~g}$ of the sample was cut into small pieces with sterilized scissors and homogenized with $225 \mathrm{~mL}$ sterile peptone water in a Stomacher ${ }^{\circledR} 400$ Circulator (Seward, Worthing, $\mathrm{UK}$ ) for $2 \mathrm{~min}$ at $230 \mathrm{rpm}$. The homogenates were subjected to serial 10-fold dilution with peptone water, and then $100 \mu \mathrm{L}$ of each dilution was spread on Bile Aesculin Azide (BAAA) agar (MERCK, 
Darmstadt, Germany) plates. After incubation at $37^{\circ} \mathrm{C}$ for $24 \mathrm{~h}$, the plates with typical numbers of colonies between 25 and 250 were selected for presumptive enumeration of Enterococcus spp. At least five presumptive enterococci colonies with typical color on BAAA agar were selected and transferred to Tryptic soy agar (TSA, Merck, Darmstadt, Germany) and were identified using biochemical testing in a VITEK ${ }^{\circledR} 2$ compact system (bioMérieux, Marcy I'Etoile, France).

\subsection{Antimicrobial Resistance}

Antimicrobial resistance of the E. faecium isolates was tested by the broth dilution method and disc diffusion method. The broth dilution test was carried out using an AST-P601 test card in a VITEK $^{\circledR} 2$ compact system (bioMérieux, Marcy I'Etoile, France) according to the manufacturer's instructions, and the disc diffusion test was performed with the antibiotic susceptibility testing disc (Oxoid Ltd., Basingstoke, UK) based on the Clinical and Laboratory Standards Institute (CLSI) standards [19]. The tested antimicrobials were as follows: ciprofloxacin, erythromycin, linezolid, teicoplanin, vancomycin, tetracycline, tigecycline, nitrofurantoin, ampicillin, amoxycillin/clavulanic acid, chloramphenicol, quinupristin/dalfopristin, clindamycin and gentamicin. The qualitative interpretation (resistant or sensitive) is based on the breakpoints for enterococci provided by the CLSI standards [19]. E. faecalis ATCC 29212 was used as a control strain to assess the quality of antimicrobial resistance testing, and all values were within the accepted limits [19].

\subsection{Rep-PCR-Based Molecular Typing}

An automated repetitive-sequence-based PCR (rep-PCR) system (DiversiLab, bioMérieux, Marcy I'Etoile, France) was used to analyze the genetic similarities of the E. faecium strains. DNA of the E. faecium was extracted using the UltraClean microbial DNA isolation Kit (Mo Bio Laboratories, Solona Beach, CA, USA) and was standardized to a concentration of ca. $25 \mathrm{ng} / \mu \mathrm{L}$. For the rep-PCR amplification of non-coding intergenic repetitive elements in the genomic DNA, the DiversiLab Enterococcus Kit (Bacterial Barcodes, Inc., Athens, GA, USA) was used according to the manufacturer's instructions. PCR conditions were as follows: initial denaturation at $94{ }^{\circ} \mathrm{C}$ for $5 \mathrm{~min} ; 30$ cycles at $94{ }^{\circ} \mathrm{C}$ for $1 \mathrm{~min}, 56^{\circ} \mathrm{C}$ for $1 \mathrm{~min}$ and $72{ }^{\circ} \mathrm{C}$ for $1 \mathrm{~min}$; with the final extension at $72{ }^{\circ} \mathrm{C}$ for $10 \mathrm{~min}$. Amplicons were analyzed using the DiversiLab system, which includes fragment separation using microfluidic chips and Agilent B2100 Bioanalyzer (Agilent Technologies Inc., Santa Clara, CA, USA). The DNA standard markers for the normalization of sample runs, and Chip kit molecular weight ladders were used. Results were analyzed using the DiversiLab software (version 3.3).

\section{Results and Discussion}

\subsection{Contamination of Enterococcus spp.}

Contamination levels of Enterococcus spp. in different types of processed pork meat products are presented in Figure 1. The mean contamination level of Enterococcus spp. was 3.0, 1.9, 1.7 and $1.6 \log \mathrm{cfu} / \mathrm{g}$ for MP, SRP, SSP and FP, respectively. The highest contamination of Enterococcus spp. was observed in MP (3.0 log cfu/g) with statistical significance compared with other types of processed pork meat products $(p<0.01)$. The contamination level of Enterococcus spp. in MP was obviously higher than SSP samples with statistical significance $(p<0.001)$. There are no statistically significant differences between SRP, SSP and FP. 


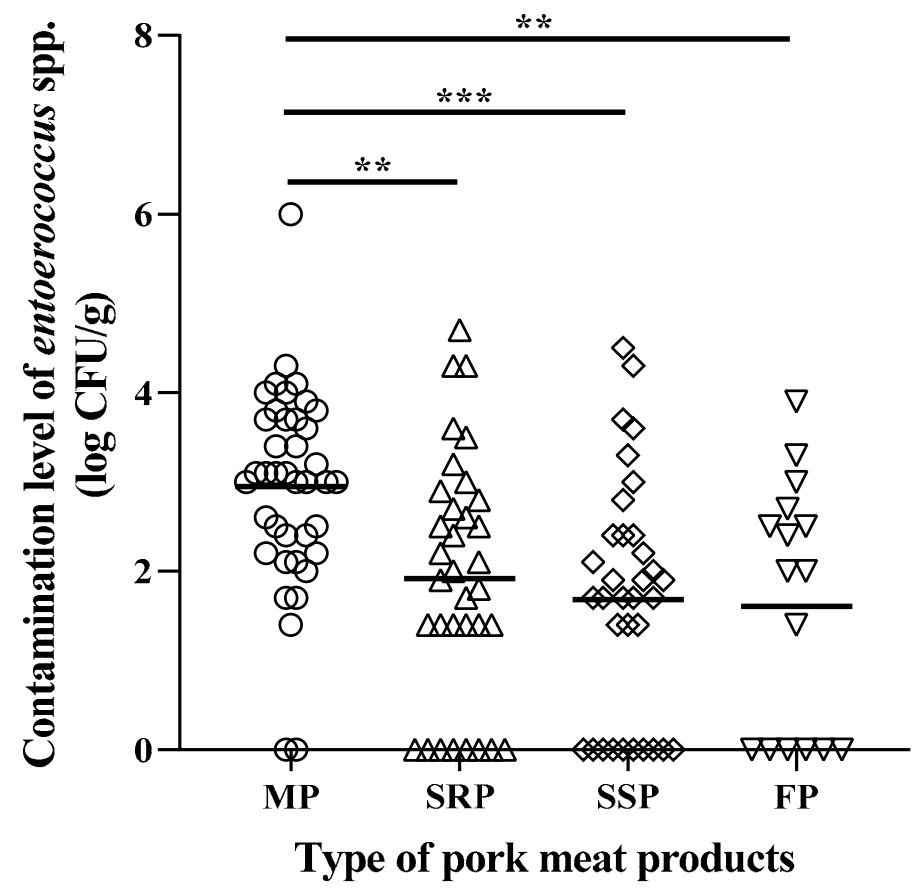

Figure 1. Contamination level of Enterococcus spp. in commercial processed pork meat products. Types of pork meat products include minced pork meat (MP), ready-to-cook pork meat products seasoned with red pepper paste (SRP), ready-to-cook pork meat products seasoned with soy sauce (SSP) and meatball-type frozen processed pork meat products (FP). Horizontal bars indicate the mean of the contamination level of enterococci of each sample. Tukey's multiple comparisons test was performed to identify the statistical significance of the contamination level of Enterococcus spp. in different types of pork meat products. ${ }^{* *}: p<0.01,{ }^{* * *}: p<0.001$.

The mean load of Enterococcus spp. observed in MP was similar to the contamination in conventional chicken carcasses $(2.9 \pm 0.4 \log \mathrm{CFU} / \mathrm{mL})$. In the same study, the contamination level of enterococci in organic chicken carcasses was $1.8 \pm 0.3 \log \mathrm{CFU} / \mathrm{mL}$ [20], which is similar to the contamination level of enterococci in SRP, SSP and FP in this study. A total of 30 E. faecium isolates were obtained from the processed pork meat products, which comprised 2 isolates from MP, 6 isolates from SRP, 2 isolates from SSP and 20 isolates from FP.

Prevalence of E. faecalis and E. faecium in the pork meat samples suggested different patterns of contamination, i.e., E. faecium was the most frequently isolated from FP followed by SRP, MP and SSP, while the highest prevalence of E. faecalis was observed in FP as well as MP, as shown in Table 1. Interestingly, the prevalence of E. faecium in the total sample was $15.3 \%$ (Table 1), but E. faecium was isolated from $68.8 \%$ of the FP samples, which suggested that some of the common ingredients might be contaminated with E. faecium, or E. faecium might be cross-contaminated from processing environments, including water. 
Table 1. Prevalence of Enterococcus faecium and Enterococcus faecalis in commercially available processed pork meat products.

\begin{tabular}{|c|c|c|c|c|}
\hline \multirow[t]{2}{*}{$\begin{array}{l}\text { Conditions at } \\
\text { Retail Market }\end{array}$} & \multirow[t]{2}{*}{ Type of Products } & \multirow[t]{2}{*}{$\begin{array}{l}\text { Isolated Enterococcus } \\
\text { spp. }\end{array}$} & \multicolumn{2}{|c|}{$\begin{array}{c}\text { Number of Positive } \\
\text { Samples/Number of Total } \\
\text { Samples (\%) }\end{array}$} \\
\hline & & & E. faecium & E. faecalis \\
\hline \multirow[t]{4}{*}{ Refrigerated } & Minced & $\begin{array}{l}\text { E. avium, E. faecalis, } \\
\text { E. faecium, E. raffinosus }\end{array}$ & $1 / 40(2.5 \%)$ & $12 / 40(30.0 \%)$ \\
\hline & $\begin{array}{l}\text { Seasoned with red } \\
\text { pepper paste }\end{array}$ & $\begin{array}{l}\text { E. avium, E. casseliflavus, } \\
\text { E. faecalis, E. faecium, } \\
\text { E. gallinarum, } \\
\text { E. raffinosus }\end{array}$ & $5 / 34(14.7 \%)$ & $5 / 34(14.7 \%)$ \\
\hline & $\begin{array}{l}\text { Seasoned with soy } \\
\text { sauce }\end{array}$ & $\begin{array}{l}\text { E. avium, E. durans, } \\
\text { E. faecalis, E. faecium, } \\
\text { E. raffinosus }\end{array}$ & $2 / 34(5.9 \%)$ & $3 / 34(8.8 \%)$ \\
\hline & Subtotal & $\begin{array}{l}\text { E. avium, E. casseliflavus, } \\
\text { E. durans, E. faecalis, } \\
\text { E. faecium, } \\
\text { E. gallinarum, } \\
\text { E. raffinosus }\end{array}$ & $8 / 108(7.4 \%)$ & $20 / 108(18.5 \%)$ \\
\hline Frozen & $\begin{array}{l}\text { Meatball-type } \\
\text { products }\end{array}$ & $\begin{array}{c}\text { E. casseliflavus, } \\
\text { E. faecalis, E. faecium }\end{array}$ & $11 / 16(68.8 \%)$ & $5 / 16(31.3 \%)$ \\
\hline Total & & $\begin{array}{l}\text { E. avium, E. casseliflavus, } \\
\text { E.durans, E. faecalis, } \\
\text { E. faecium, } \\
\text { E. gallinarum, } \\
\text { E. raffinosus }\end{array}$ & 19/124 (15.3\%) & $25 / 124(20.2 \%)$ \\
\hline
\end{tabular}

Other enterococci, including E. avium, E. raffinose, E. casseliflavus, E. gallinarum, E. durans, were also isolated from processed pork meat products, which is supported by the previous study on the isolation of enterococci, i.e., Enterococcus spp., including E. hirae, E. casselifavus, E. durans, E. devriesei, E. gilvus, E. mundtii, and E. thailandicus, which were also isolated from beef and pork samples [11]. SRP and SSP contained a variety of ingredients including seasoning and fresh vegetables such as garlic, green onion and onions, and thus, different enterococci might originate from seasoning and raw vegetables. FP also contains different types of seasoning and vegetables and even chicken or beef as ingredients, but relatively less diverse enterococci, including E. casseliflavus, E. faecalis and E. faecium were isolated.

In this study, E. faecium and E. faecalis were observed in $15.3 \%$ and $20.2 \%$ of the processed pork meat products, while a previous study suggested that E. faecium and E. faecalis was detected in $11.3 \%$ and $69.5 \%$ of red meat [11]. In Argentinean artisanal dry fermented sausages, the most frequently isolated enterococci was E. faecium (56\%), followed by E. faecalis (17\%) and other species (E. durans, E. casseliflavus, and E. mundtii) [21].

\subsection{Antimicrobial Resistance}

The antimicrobial resistance of $30 \mathrm{E}$. faecium isolates were analyzed against fourteen antimicrobials belonging to 12 different antimicrobial classes (Table 2). As indicated, 9 out of the 14 tested antimicrobials were critically important antimicrobials for human medicine, and the remaining were highly important antimicrobials for human medicine, as categorized by WHO AGISAR [22]. 
Table 2. Antimicrobial resistance of Enterococcus faecium and Enterococcus faecalis against 14 antibiotics.

\begin{tabular}{cccccccc}
\hline \multirow{2}{*}{$\begin{array}{c}\text { Antimicrobial } \\
\text { Class }\end{array}$} & \multirow{2}{*}{ Antimicrobials } & \multicolumn{2}{c}{ No. of E. faecium Isolates (\%) } & \multicolumn{2}{c}{ No. of E. faecalis Isolates (\%) } \\
\cline { 3 - 7 } & & $\mathbf{R}^{\mathbf{a}}$ & $\mathbf{I}$ & $\mathbf{S}$ & $\mathbf{R}$ & $\mathbf{I}$ & $\mathbf{S}$ \\
\hline Quinolones & Ciprofloxacin & $0(0.0)$ & $4(13.3)$ & $26(86.6)$ & $0(0.0)^{\mathrm{b}}$ & $4(11.1)$ & $32(88.9)$ \\
\hline $\begin{array}{c}\text { Macrolides and } \\
\text { ketolides }\end{array}$ & Erythromycin & $24(80.0)$ & $5(16.7)$ & $1(3.3)$ & $4(11.1)^{\mathrm{b}}$ & $14(38.9)$ & $18(50.0)$ \\
\hline Oxazolidinones & Linezolid & $0(0.0)$ & $0(0.0)$ & $30(100.0)$ & $0(0.0)^{\mathrm{b}}$ & $1(2.8)$ & $35(97.2)$ \\
\hline Nitrofurantoins & Nitrofurantoin & $6(20.0)$ & $20(66.6)$ & $4(13.3)$ & $1(2.8)^{\mathrm{b}}$ & $8(22.2)$ & $27(75.0)$ \\
\hline Glycopeptides & Teicoplanin & $0(0.0)$ & $0(0.0)$ & $30(100.0)$ & $0(0.0)^{\mathrm{b}}$ & $0(0.0)$ & $36(100.0)$ \\
\hline Glycopeptides & Vancomycin & $0(0.0)$ & $0(0.0)$ & $30(100.0)$ & $0(0.0)^{\mathrm{b}}$ & $0(0.0)$ & $36(100.0)$ \\
\hline Tetracyclines & Tetracycline & $3(10.0)$ & $0(0.0)$ & $27(90.0)$ & $21(58.3)^{\mathrm{b}}$ & $0(0.0)$ & $15(41.7)$ \\
\hline Glycylcyclines & Tigecycline & $0(0.0)$ & $0(0.0)$ & $30(100.0)$ & $0(0.0)^{\mathrm{b}}$ & $0(0.0)$ & $36(100.0)$ \\
\hline Penicillins & Amoxycillin/clavulanic & $0(0.0)$ & $0(0.0)$ & $30(100.0)$ & $0(0.0)^{\mathrm{b}}$ & $0(0.0)$ & $36(100.0)$ \\
\hline acid & Aampicillin & $0(0.0)$ & $0(0.0)$ & $30(100.0)$ & $0(0.0)^{\mathrm{b}}$ & $0(0.0)$ & $36(100.0)$ \\
\hline Amphillin & Chloramphenicol & $1(3.3)$ & $1(3.3)$ & $28(93.3)$ & $0(0.0)^{\mathrm{b}}$ & $0(0.0)$ & $36(100.0)$ \\
\hline Streptogramins & Quinupristin/dalfopristin & $0(0.0)$ & $0(0.0)$ & $30(100.0)$ & $25(69.4)$ & $6(16.7)$ & $5(13.9)$ \\
\hline Lincosamides & Clindamycin & $15(50.0)$ & $8(26.6)$ & $7(23.3)$ & $32(88.9)$ & $0(0.0)$ & $4(11.1)$ \\
\hline Aminoglycosides & Gentamicin & $1(3.3)$ & $0(0.0)$ & $29(96.7)$ & $0(0.0)$ & $0(0.0)$ & $36(100.0)$ \\
\hline R, resistant & $\mathrm{I}$, & in &
\end{tabular}

${ }^{a} \mathrm{R}$, resistant; $\mathrm{I}$, intermediate resistance; and $\mathrm{S}$, sensitive to the antimicrobials. ${ }^{\mathrm{b}}$ Antimicrobial resistance of E. faecalis against ciprofloxacin, erythromycin, linezolid, nitrofurantoin, teicoplanin, vancomycin, tetracycline, tigecycline, amoxycillin/clavulanic acid, ampicillin and chloramphenicol were adopted from our previous study for comparison with antimicrobial resistance of E. faecium [23]. Antimicrobial resistance of the 36 E. faecalis against quinupristin/dalfopristin, clindamycin and gentamicin were analyzed in the present study.

As shown in Table 2, the highest incidence of antimicrobial resistance was observed for erythromycin (80\% resistance) followed by clindamycin $(50 \%)$ and nitrofurantoin $(20 \%)$ in the case of E. faecium isolates. Resistance and intermediate resistance to erythromycin, nitrofurantoin and clindamycin was detected in $96.7 \%, 86.6 \%$ and $76.6 \%$, respectively. Because erythromycin is considered to be a critically important antimicrobial for human use, high antimicrobial resistance against erythromycin poses a public health problem. Nitrofurantoin is used to treat urinary tract infections caused by Enterococcus spp., including vancomycin-resistant enterococci (VRE) [1]. The high prevalence of resistant $(20 \%)$ and intermediate resistant (66.6\%) E. faecium against nitrofurantoin in pork meat products suggested that special consideration of the prevalence and mitigation of the spread of antimicrobial-resistant E. faecium in the food system is needed. All the tested E. faecium isolates were susceptible to vancomycin and teicoplanin in this study. No E. faecium isolates were resistant or intermediate resistant to quinupristin/dalfopristin in our study, although enterococci are known as intrinsically resistant to quinupristin/dalfopristin [11].

To compare with the antimicrobial resistance patterns of E. faecium, information on the antimicrobial resistance of $E$. faecalis against 11 antimicrobials were adopted from our previous report [23]. Resistance against quinupristin/dalfopristin, clindamycin and gentamicin were further analyzed for 36 E. faecalis isolates in this study. The highest resistance of E. faecalis was observed for clindamycin (88.9\%) followed by quinupristin/dalfopristin (69.4\%) and tetracycline (58.3\%). Obviously, the high incidence of E. faecalis isolates resistant to clindamycin and quinupristin/dalfopristin seems related to intrinsically resistant characteristics of enterococci against clindamycin and quinupristin/dalfopristin [1,24]. In the case of E. faecalis isolated from processed pork meat products, a higher incidence of resistant isolates was observed for tetracycline (58.3\%), quinupristin/dalfopristin (69.4\%) and clindamycin (88.9\%) compared to E. faecium. Both the E. faecium and E. faecalis strains are not resistant to vancomycin, ciprofloxacin, linezolid, teicoplanin, tigecycline, amoxycillin/clavulanic acid and ampicillin, which are critically important antimicrobials for human use (Table 2).

National surveillance data on the antimicrobial resistance of E. faecium isolated from livestock and livestock products in Korea showed that the highest antimicrobial resistance of E. faecium 
$(n=197)$ isolated from pig feces was observed for erythromycin $(48.2 \%)$ followed by tigecycline $(17.3 \%)$ and tetracycline (16.2\%) [25]. Generally, E. faecium isolated from pig feces showed similar or higher antimicrobial resistance than antimicrobial resistances observed in this study, except for erythromycin [25]. The highest resistance of E. faecium $(n=14)$ isolated from domestic produced pork meat products was observed for tetracycline (42.9\%) followed by quinupristin/dalfopristin $(35.7 \%)$, erythromycin $(14.3 \%)$ and clindamycin $(14.3 \%)$. In case of E. faecium $(n=9)$ isolated from imported pork meat products, the most frequently observed antimicrobial resistances were quinupristin/dalfopristin (55.6\%) and tetracycline (22.2\%) [25]. No VRE were isolated from pig feces and pork meat products in the previous study [25]. According to a report from the Korean global antimicrobial resistance surveillance system (Kor-GLASS) for 2017, resistance to vancomycin $(34.0 \%, 98 / 288)$ and teicoplanin $(18.8 \%, 98 / 288)$ was frequently observed in E. faecium strains isolated from human [26]. Resistance to erythromycin was not provided in Kor-GLASS [26]. However, another report on the antimicrobial resistance of E. faecium human isolates ( $n=21$ ) obtained from the Asian Bacterial Bank of the Asia Pacific Foundation for Infectious Diseases showed that antimicrobial resistance to ampicillin, ciprofloxacin, erythromycin and tetracycline was $100 \%, 100 \%, 95.2 \%$ and $33.3 \%$, respectively [27].

Similar to this study, no VRE were isolated from chicken carcasses in Korea [20]. However, 8 fecal and 3 produce isolates out of 389 Enterococcus spp. isolates resistant to vancomycin and teicoplanin were reported in Korea [28]. The previously reported VRE isolates showed multidrug-resistant properties against ampicillin, penicillin, erythromycin, rifampin, gentamicin, teicoplanin, vancomycin, streptomycin, etc. VRE isolates from fresh produce seemed isolated from humans because the isolates from fresh produce were shown to have common shared characteristics with the isolates from humans, such as antimicrobial resistance, multilocus sequence typing (MLST), and Tn 1546 transposon analysis [28].

Enterococci are intrinsically susceptible to tetracyclines and erythromycin, although acquired resistance to these agents is widespread (except for tigecycline) [24]. We observed $80.0 \%$ and $11.1 \%$ resistance against erythromycin for E. faecium and E. faecalis isolated from processed pork meat products, respectively. In the previous report in Korea, $17.3-44.2 \%$ of the E. faecalis isolates obtained from chicken carcasses showed resistance to erythromycin [20]. We also observed that $10 \%$ and $58.3 \%$ of the E. faecium and E. faecalis strains are resistant against tetracycline, which is considered a highly important antimicrobial for human medicine (Table 2). The newer agents linezolid, tedizolid, daptomycin, televancin, and oritavancin are active against enterococci, and the pristinamycin combination quinupristin/dalfopristin is active against E. faecium only [24]. In this study, no resistance against linezolid was observed for E. faecium and E. faecalis except one intermediate resistant E. faecalis to linezolid.

Varied uses of antimicrobials as animal medicines might cause the different antimicrobial resistance patterns of pathogens. For example, incidences of resistance against linezolid, tigecycline and vancomycin were less than $1 \%$ in any species or meat source, and the low incidences can be explained by these antimicrobials not being used in food animals. In contrast, tetracyclines are the most heavily used antimicrobials in food animals and they revealed the greatest resistance prevalence [1]. In Korea, tetracyclines, followed by penicillins, were the most frequently sold antimicrobials for veterinary use [29]. The highest resistance rate against tetracycline of E. faecalis might be related to the amount of antimicrobials sold in Korea. No VRE observed in this study could be explained by the banning of the use of avoparcin in Korea in 1997. The use of animal growth promoters such as avoparcin is reportedly associated with the appearance of VRE [21,25]. However, in the case of E. faecium, only $10 \%$ of the isolates possess resistance against tetracycline. Similarly, differences in resistance prevalence between E. faecalis and E. faecium isolated from retail meat in the United States with statistical significance were observed for tetracycline, gentamycin, erythoromycin, nitrofurantoin, ciprofloxacin and quinupristin/dalfopristin [1].

Among 30 tested E. faecium, only 4 isolates were susceptible to all 14 antimicrobials examined. Some of the E. faecium isolates (23.3\%) were resistant to single antimicrobials (Table 3). Multi-drug 
resistances (MDR) were observed for $13.3 \%$ of the tested E. faecium isolates. E. faecium isolated from SRP showed the highest incidence of MDR followed by isolates from FP. All of the MDR isolates are resistant to clindamycin. Considering that E. faecium are naturally resistant to clindamycin, the incidence of MDR E. faecium obtained from processed pork meat produces is quite low. Except for clindamycin, the most frequently observed resistance patterns against two antimicrobials were erythromycin-nitrofurantoin followed by erythromycin-tetracycline.

Table 3. Multidrug resistance of Enterococcus faecium isolated from processed pork meat products.

\begin{tabular}{cccccc}
\hline \multirow{2}{*}{$\begin{array}{c}\text { Resistant } \\
\text { Antimicrobials }\end{array}$} & \multicolumn{4}{c}{ Number of Strains (\%) for Different Types of Pork Meat Products } \\
\cline { 2 - 6 } & MP $^{b}$ & SRP & SSP & FP & Total \\
\hline None & $0(0.0)$ & $0(0.0)$ & $2(6.7)$ & $2(6.7)$ & $4(13.3)$ \\
\hline CHA $^{a}$ & $1(3.3)$ & $0(0.0)$ & $0(0.0)$ & $0(0.0)$ & $1(3.3)$ \\
GM & $0(0.0)$ & $0(0.0)$ & $0(0.0)$ & $0(0.0)$ & $0(0.0)$ \\
E & $0(0.0)$ & $1(3.3)$ & $0(0.0)$ & $5(16.7)$ & $6(20.0)$ \\
FT & $0(0.0)$ & $0(0.0)$ & $0(0.0)$ & $0(0.0)$ & $0(0.0)$ \\
\hline GM, CM & $1(3.3)$ & $0(0.0)$ & $0(0.0)$ & $0(0.0)$ & $1(3.3)$ \\
E, CM & $0(0.0)$ & $2(6.7)$ & $0(0.0)$ & $7(23.3)$ & $9(30.0)$ \\
E, FT & $0(0.0)$ & $0(0.0)$ & $0(0.0)$ & $4(13.3)$ & $4(13.3)$ \\
CM, FT & $0(0.0)$ & $0(0.0)$ & $0(0.0)$ & $1(3.3)$ & $1(3.3)$ \\
E, TE & $0(0.0)$ & $0(0.0)$ & $0(0.0)$ & $0(0.0)$ & $0(00.0)$ \\
\hline E, TE, CM & $0(0.0)$ & $3(10.0)$ & $0(0.0)$ & $0(0.0)$ & $3(10.0)$ \\
E, CM, FT & $0(0.0)$ & $0(0.0)$ & $0(0.0)$ & $1(3.3)$ & $1(3.3)$ \\
\hline
\end{tabular}

Antimicrobial resistance of 30 E. faecium isolates were tested against 14 antimicrobials, including gentamicin, ciprofloxacin, tetracycline, ampicillin, chloramphenicol, erythromycin, amoxycillin/clavulanic acid, clindamycin, quinupristin/dalfopristin, linezolid, teicoplanin, vancomycin, tigecycline and nitrofurantoin. ${ }^{a} \mathrm{CHA}$ : chloramphenicol, GM: gentamicin, E, erythromycin; FT, nitrofurantoin; TE, tetracycline. CM: clindamycin. ${ }^{b}$ Each abbreviation represents the type of product as follows: MP: minced pork meat, SRP: ready-to-cook pork meat seasoned with red pepper paste, SSP: ready-to-cook pork meat seasoned with soy sauce, FP: frozen meatball-type processed pork meat products.

According to the population-weighted consumption and distribution of antimicrobial agents, the most frequently used for humans were penicillins, followed by 2nd generation cephalosporins, macrolides, and fluoroquinolones, which have 4.52, 4.47, 3.32, and 2.75 defined daily dose per 1000 people per day, respectively in Korea [30]. In the present study, there was no observed resistance against penicillins for E. faecium and E. faecalis; however, the high resistance of E. faecium (80\%) against erythromycin suggested that risk management for antimicrobial resistance is needed, considering the importance of antimicrobials for human use and possible cross-contamination via food $[4,5]$.

\subsection{Analysis of Strain Relatedness by Rep-PCR}

To identify the strain relatedness of E. faecium isolates obtained from different types of processed pork meat products, molecular typing was performed with Diversilab, an automated rep-PCR method. Initial analysis of rep-PCR dendrograms showed two clusters (C1 and C2) with a similarity $\geq 95 \%$ and 6 singleton isolates in Figure 2. In the cluster C1, distinctive sub-clusters with a similarity $\geq 98 \%$ were detected as C1-1 and C1-2. Surprisingly, 18 out of the 20 E. faecium isolates originated from FP were clustered as C1-2 and most of the E. faecium isolates clustered as C1-2 were resistant to erythromycin (Figure 2). Rep-PCR typing using Diversilab has value as a semi-automated, very quick, certified genotyping method using a centralized and standardized analysis software. Thus, it can be implemented in a routine diagnostic laboratory workflow [31]. Various molecular typing methods are used for the surveillance of foodborne pathogens and outbreak investigations, differing widely in information content and discriminatory power. Presently, the focus is shifting to whole genome sequencing (WGS) as an analytical tool [32]. In this study, we only provided the rep-PCR-based molecular typing E. faecium, but additional molecular or phenotypic analysis including Pulsed field gel electrophoresis (PFGE), MLST and WGS is recommended to further elucidate the rep-PCR typing result. 


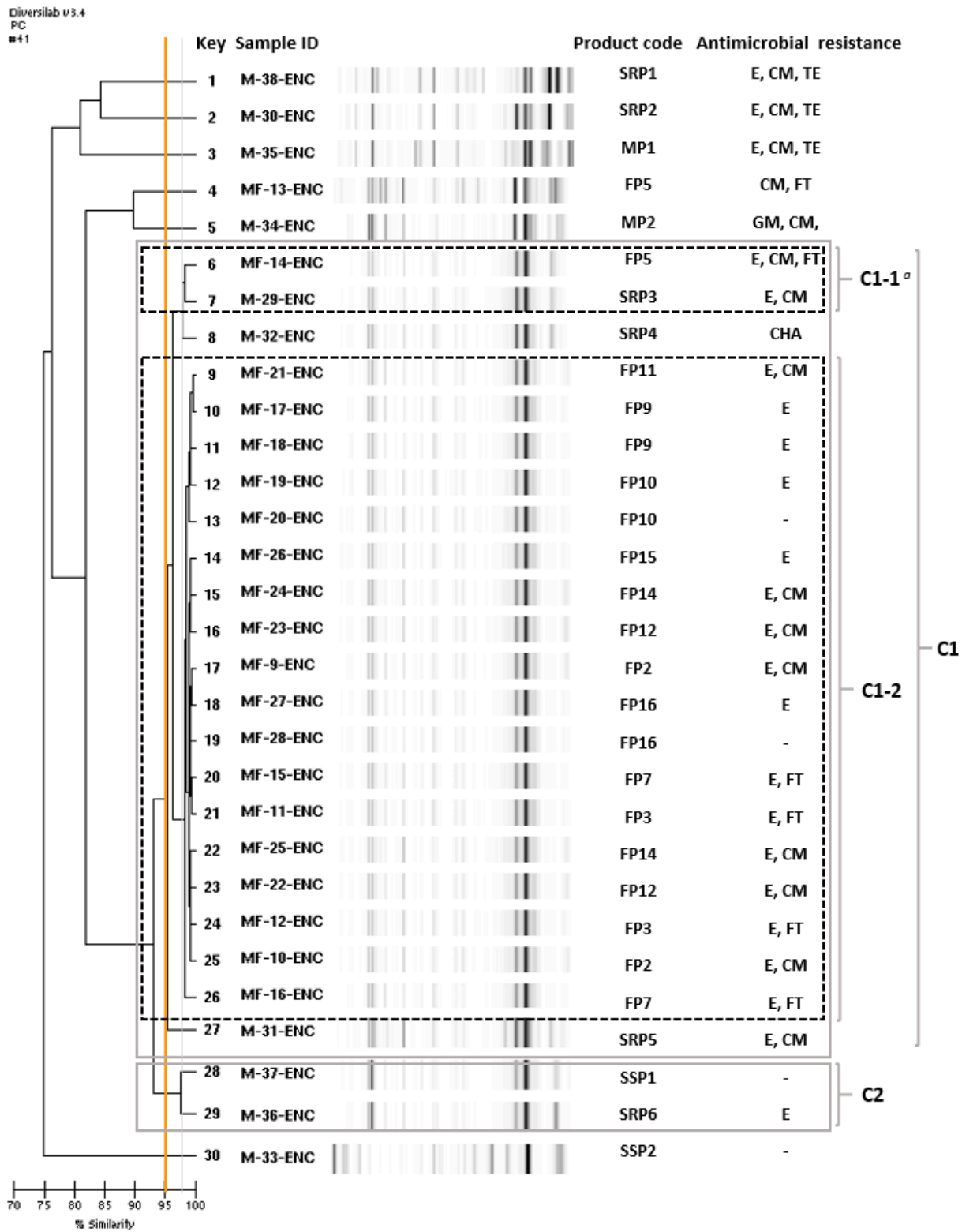

Figure 2. Molecular typing of Enterococcus faecium isolates obtained from the commercial processed pork meat products in Korea. The dendrogram shows the molecular typing results obtained by rep-PCR with the Diversilab method. The total number of isolates analyzed in this study was 30 . Clusters with Diversilab similarity $\geq 95 \%$ are marked with a grey rectangle and numbered (C1 and C2). Clusters with a similarity $\geq 98 \%$ are marked with a black dotted line and numbered C1-1 and C1-2. The abbreviation in the product code represents MP (minced pork meat), SRP (ready-to-cook pork meat products seasoned with red pepper paste), SSP (ready-to-cook pork meat products seasoned with soy sauce) and FP (frozen meatball-type processed pork meat products). Antimicrobial resistances were observed for Erythromycin (E), Clindamycin (CM), Tetracycline (TE), Nitrofurantoin (FT), Chloramphenicol (CHA) and Gentamicin (GM). ${ }^{a}$ Cluster number.

The high strain relatedness of E. faecium isolated from FP products might be explained by: (1) E. faecium isolates might be contaminated in the common ingredients used in the varieties of FP products analyzed in this study, or (2) E. faecium isolates might be cross-contaminated from the processing environment including water of the manufacturing company. To prove the first assumption, i.e., E. faecium isolates might originate from ingredients of FP products, sources and content of the meat 
used as main ingredients together with information on the product name and manufacturing company are compared.

As in Table 4, all the FP products contained domestically produced pork meat except for one sample (Key No. 21), but contents of the pork meats were different (33.3 75.2\%) according to the product type. Other types of processed pork meat products (M, SRP, SS) also contained domestically produced pork meat.

Table 4. Information on the pork meat products analyzed for E. faecium.

\begin{tabular}{|c|c|c|c|}
\hline Key No. ${ }^{a}$ & Product Code & Manufacturing Company & Main Ingredients \\
\hline 1 & SRP1 & $\mathrm{A}^{b}$ & Pork \\
\hline 2 & SRP2 & $\mathrm{B}$ & Pork \\
\hline 3 & MP1 & $\mathrm{C}$ & Pork \\
\hline 4 & FP5 & $\mathrm{D}$ & Pork (66.03\%), Chicken (4.4\%) \\
\hline 5 & $\mathrm{MP2}$ & $\mathrm{E}$ & Pork $100 \%$ \\
\hline 6 & FP5 & $\mathrm{D}$ & Pork $(66.03 \%)$, Chicken $(4.4 \%)$ \\
\hline 7 & SRP3 & A & Pork meat \\
\hline 8 & SRP4 & $\mathrm{F}$ & Pork meat \\
\hline 9 & FP11 & G & Pork $(20.56 \%)$, chicken $(38.16 \%)$ \\
\hline 10 & FP9 & $\mathrm{H}$ & Pork $(75.2 \%)$ \\
\hline 11 & FP9 & $\mathrm{H}$ & Pork $(75.2 \%)$ \\
\hline 12 & FP10 & $\mathrm{H}$ & Pork $(60.94 \%)$, chicken \\
\hline 13 & FP10 & $\mathrm{H}$ & Pork $(60.94 \%)$, chicken \\
\hline 14 & FP15 & I & $\begin{array}{l}\text { Pork }(30.6 \%) \text {, chicken }(22.95 \%) \text {, beef } \\
(7.65 \%)\end{array}$ \\
\hline 15 & FP14 & $\mathrm{J}$ & Pork $(29.18 \%)$, chicken $(20.84 \%)$ \\
\hline 16 & FP12 & G & Pork (50.74\%), Chicken (19.73\%) \\
\hline 17 & FP2 & G & Pork (62.22\%), Chicken (6.89\%) \\
\hline 18 & FP16 & K & Pork $(78.43 \%)$ \\
\hline 19 & FP16 & K & Pork $(78.43 \%)$ \\
\hline 20 & FP7 & $\mathrm{H}$ & Pork (33.37\%), chicken (12.05\%) \\
\hline 21 & FP3 & $\mathrm{L}$ & Pork (domestic 59.2\%, imported 14.8\%) \\
\hline 22 & FP14 & $\mathrm{J}$ & Pork (29.18\%), chicken $(20.84 \%)$ \\
\hline 23 & FP12 & G & Pork (50.74\%), Chicken (19.73\%) \\
\hline 24 & FP3 & $\mathrm{L}$ & Pork (domestic 59.2\%, imported $14.8 \%$ ) \\
\hline 25 & FP2 & G & Pork (62.22\%), Chicken (6.89\%) \\
\hline 26 & FP7 & $\mathrm{H}$ & Pork $(33.37 \%)$, chicken $(12.05 \%)$ \\
\hline 27 & SRP5 & $\mathrm{F}$ & Pork \\
\hline 28 & SSP1 & M & Pork $(70 \%)$ \\
\hline 29 & SRP6 & $\mathrm{N}$ & Pork $(70 \%)$ \\
\hline 30 & SSP2 & $\mathrm{N}$ & Pork (70\%) \\
\hline
\end{tabular}

${ }^{a}$ Key number are adopted from rep-PCR data in Figure 2. ${ }^{b}$ Locations of the manufacturing company were presented in Figure 3. All the pork meat used as the main ingredient was domestically grown, except for FP3 (key number 21). 
Chicken meat is also frequently used as an ingredient of FP products: 16 out of 20 samples of FP products contained chicken meat as ingredients. However, E. faecium strains originated from FP products containing pork meat only (Key No. 10, 11, 18, 19) showed high similarity ( $\geq 98 \%$ ) with E. faecium isolated from FP products containing chicken and pork meat (Key No. 4, 6, 9, 12-17, 20-26) as shown in Figure 2 and Table 4. Therefore, E. faecium clustered as C1-2 might originate from ingredients other than pork and chicken meat. Most of the FP products contain seasoning and vegetables such as green onion but no information on the content of the vegetables or details of seasoning was provided in the food label of FP products. To identify the exact sources of E. faecium contamination, more detailed information on the minor ingredients, for example, seasoning, are needed, and prevalence of enterococci in the ingredients and molecular typing of the enterococci isolates should be analyzed.

For the second assumption, that the E. faecium isolates might originate from processing environments or water, the best way to prove the hypothesis is to analyze the occurrence of E. faecium in the processing environments including water [33]. However, we were not able to access the processing environments of the manufacturing companies of FP products to monitor the occurrence of enterococci, unfortunately. Instead, manufacturing companies of FP products and locations of each company were analyzed as an indirect approach. As shown in Table 4 and Figure 3, FP products analyzed in this study were manufactured at seven different companies (Company D, G, H, I, J, K and L) located in scattered areas of Korea. The closely related E. faecium isolates originated from FP products manufactured at seven companies with different geographical locations, suggesting very low possibilities of cross-contamination of E. faecium from processing environments.

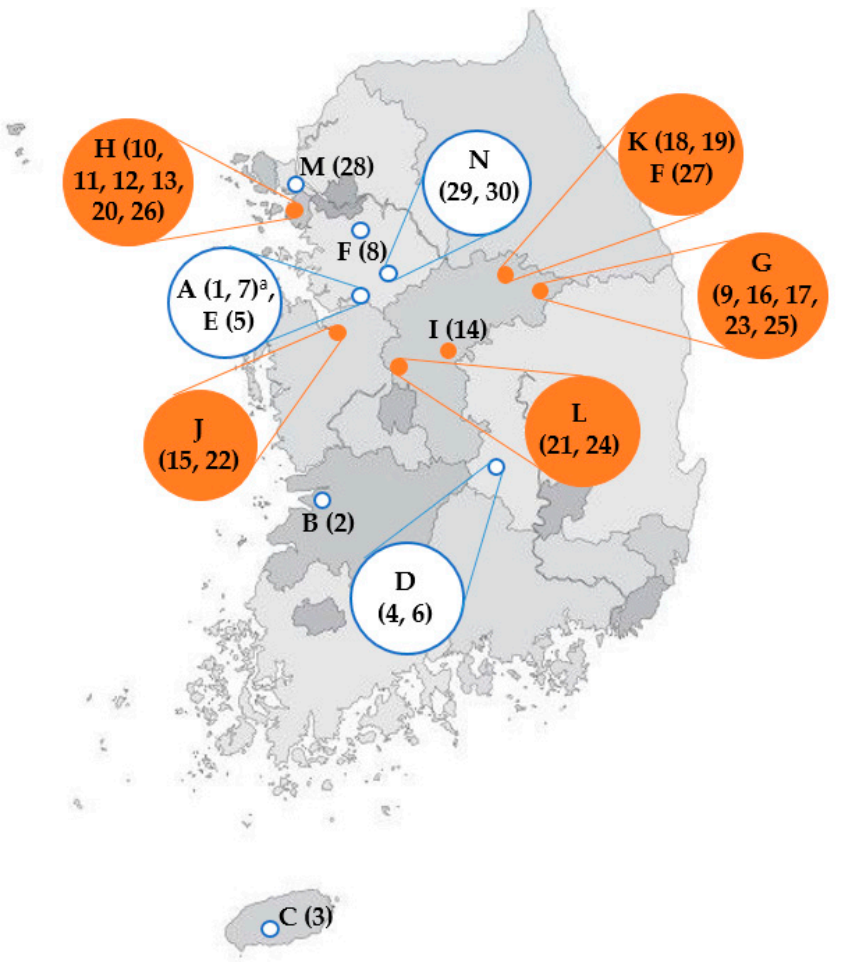

Figure 3. Location of processing company for pork meat products analyzed for E. faecium contaminations. Orange closed circles and blue open circles represent the geographical position of processing company produced samples analyzed in this study. Orange closed circles indicate the geographical position of processing company produced samples contaminated with closely related E. faecium with a similarity $\geq 98 \%$, which were analyzed by automated rep-PCR in Figure 2. a A (1, 7): Alphabet indicates the processing company. Numbers in parentheses depict the key number in the dendrogram of rep-PCR and Table 4. 
In the food supply chain, the pathogenic potential of enterococci is of concern due to their ability to harbor and transfer virulence and antimicrobial resistance to other pathogens based on horizontal gene transfer. Although E. faecium tested in this study have low MDR, $85 \%$ of the E. faecium isolated from FP showed erythromycin resistance (Figure 2), which is categorized as a critically important antimicrobial for human medicine [22]. Because spread of antimicrobial resistance, especially community-acquired antimicrobial resistance, is increasing and food can be a good source of contamination for humans, early detection of the transmission of antimicrobial-resistant enterococci in the food chain is important to control antimicrobial resistance due to food and food environments [34].

\section{Conclusions}

In conclusion, contamination levels of Enterococcus spp. were analyzed in processed pork meat products $(n=124)$ commercially available in Korea. We found that contamination in the MP is the highest followed by SRP and SSP. For the 30 E. faecium isolates, antimicrobial resistance was analyzed against 14 antimicrobials belonging to 12 antimicrobial classes. The highest incidence of antimicrobial-resistant E. faecium was observed for erythromycin ( $80 \%$ resistance) followed by clindamycin (50\%) and nitrofurantoin (20\%). No vancomycin-resistant enterococci were observed. Rep-PCR showed distinctive clusters with a similarity $\geq 98 \%$ consisting of 18 E. faecium isolates from FP manufactured in seven companies, suggesting that $E$. faecium isolates obtained from FP may be closely related. The results from Rep-PCR would reflect the real relatedness of E. faecium isolates, but additional molecular or phenotypic analysis including PFGE, MLST and WGS is recommended to further elucidate the rep-PCR typing result. High prevalence of erythromycin-resistant E. faecium among the clustered enterococci suggested that antimicrobial-resistant enterococci might be transmitted via food and cause food safety and public health concerns. Information on the contamination and antimicrobial resistance patterns combined with molecular typing analyzed in this study can be useful to derive risk management options, preventing the spread of antimicrobial-resistant enterococci in food.

Author Contributions: Conceptualization, H.J.K.; methodology, M.K.; formal analysis and investigation, H.J.K. and M.K.; writing—original draft preparation, review and editing, H.J.K.; funding acquisition, H.J.K. All authors have read and agreed to the published version of the manuscript.

Funding: This research was funded by a grant from Korea Food and Drug Administration in 2010 and by Korea Food Research Institute, grant number E0192101-02.

Acknowledgments: The authors are appreciated with technical support provided by A.R. Jeong.

Conflicts of Interest: The authors declare no conflict of interest.

\section{References}

1. Tyson, G.H.; Nyirabahizi, E.; Crarey, E.; Kabera, C.; Lam, C.; Rice-Trujillo, C.; McDermott, P.F.; Tate, H. Prevalence and antimicrobial resistance of enterococci isolated from retail meats in the United States, 2002 to 2014. Appl. Environ. Microbiol. 2018, 84,1-9. [CrossRef]

2. Kelesidis, T.; Humphries, R.; Uslan, D.Z.; Pegues, D.A. Daptomycin nonsusceptible enterococci: An emerging challenge for clinicians. Clin. Infect. Dis. 2011, 52, 228-234. [CrossRef] [PubMed]

3. Pfaller, M.A.; Cormican, M.; Flamm, R.K.; Mendes, R.E.; Jones, R.N. Temporal and geographic variation in antimicrobial susceptibility and resistance patterns of enterococci: Results from the SENTRY Antimicrobial Surveillance Program, 1997-2016. Open Forum Infect. Dis. 2019, 6, S54-S62. [CrossRef] [PubMed]

4. Franz, C.M.A.P.; Holzapfel, W.H.; Stiles, M.E. Enterococci at the crossroads of food safety? Int. J. Food Microbiol. 1999, 47, 1-24. [CrossRef]

5. Franz, C.M.A.P.; Stiles, M.E.; Schleifer, K.H.; Holzapfel, W.H. Enterococci in foods-A conundrum for food safety. Int. J. Food Microbiol. 2003, 88, 105-122. [CrossRef]

6. Woodford, N.; Livermore, D.M. Infections caused by gram-positive bacteria: A review of the global challenge. J. Infect. 2009, 59, S4-S16. [CrossRef] 
7. Billington, E.O.; Phang, S.H.; Gregson, D.B.; Pitout, J.D.D.; Ross, T.; Church, D.L.; Laupland, K.B.; Parkins, M.D. Incidence, risk factors, and outcomes for Enterococcus spp. blood stream infections: A population-based study. Int. J. Infect. Dis. 2014, 26, 76-82. [CrossRef]

8. Hasan, K.A.; Ali, S.A.; Rehman, M.; Bin-Asif, H.; Zahid, S. The unravelled Enterococcus faecalis zoonotic superbugs: Emerging multiple resistant and virulent lineages isolated from poultry environment. Zoonoses Public Health 2018, 65, 921-935. [CrossRef]

9. Weiss, A.; Domig, K.J.; Kneifel, W. Comparison of selective media for the enumeration of probiotic enterococci from animal feed. Food Technol. Biotechnol. 2005, 43, 147-155.

10. MuÈller, T.; Ulrich, A.; Ott, E.-M.; MuÈller, M. Identification of plant-associated enterococci. J. Appl. Microbiol. 2001, 91, 268-278.

11. Golob, M.; Pate, M.; Kušar, D.; Dermota, U.; Avberšek, J.; Papić, B.; Zdovc, I.; Bondi, M. Antimicrobial Resistance and Virulence Genes in Enterococcus faecium and Enterococcus faecalis from Humans and Retail Red Meat. Biomed Res. Int. 2019, 2019, 14-16. [CrossRef] [PubMed]

12. Jones, M.E.; Draghi, D.C.; Thornsberry, C.; Karlowsky, J.A.; Sahm, D.F.; Wenzel, R.P. Emerging resistance among bacterial pathogens in the intensive care unit-A European and North American surveillance study (2000-2002). Ann. Clin. Microbiol. Antimicrob. 2004, 3, 1-11. [CrossRef]

13. Willems, R.J.; van Schaik, W. Transition of Enterococcus faecium from commensal organism to nosocomial pathogen. Future Microbiol. 2009, 4, 1125-1135. [CrossRef] [PubMed]

14. Bortolaia, V.; Espinosa-Gongora, C.; Guardabassi, L. Human health risks associated with antimicrobial-resistant enterococci and Staphylococcus aureus on poultry meat. Clin. Microbiol. Infect. 2016, 22, 130-140. [CrossRef]

15. Miller, W.R.; Munita, J.M.; Arias, C.A. Mechanisms of antibiotic resistance in enterococci. Expert Rev. Anti-Infect. Ther. 2014, 12, 1221-1236. [CrossRef]

16. Kim, H.J.; Griffiths, M.W.; Fazil, A.M.; Lammerding, A.M. Probabilistic risk model for staphylococcal intoxication from pork-based food dishes prepared in food service establishments in korea. J. Food Prot. 2009, 72, 1897-1908. [CrossRef]

17. Kim, Y.B.; Seo, H.J.; Seo, K.W.; Jeon, H.Y.; Kim, D.K.; Kim, S.W.; Lim, S.K.; Lee, Y.J. Characteristics of high-Level ciprofloxacin-Resistant Enterococcus faecalis and Enterococcus faecium from retail chicken meat in Korea. J. Food Prot. 2018, 81, 1357-1363. [CrossRef]

18. International Organization for Standardization (IOS). Water Quality-Detection and Enumeration of Intestinal Enterococci in Surface and Waste Water. Part I: Miniaturized Method (Most Probable Number) by Inoculation in Liquid Mediumitle; I1998, ISO 7899-1; International Organization for Standardization: Geneva, Switzerland, 2000.

19. Clinical and Laboratory Standards Institute (CLSI). Performance Standards for Antimicrobial Susceptibility Testing; Twentieth informational supplement; CLSI document M100-S20; Clinical and Laboratory Standards Institute: Wayne, PA, USA, 2010.

20. Kim, Y.J.; Park, J.H.; Seo, K.H. Comparison of the loads and antibiotic-resistance profiles of Enterococcus species from conventional and organic chicken carcasses in South Korea. Poult. Sci. 2018, 97, 271-278. [CrossRef]

21. Fontana, C.; Gazzola, S.; Cocconcelli, P.S.; Vignolo, G. Population structure and safety aspects of Enterococcus strains isolated from artisanal dry fermented sausages produced in Argentina. Lett. Appl. Microbiol. 2009, 49, 411-414. [CrossRef]

22. WHO Advisory Group on Integrated Surveillance of Antimicrobial Resistance (AGISAR). Critically Important Antimicrobials for Human Medicine, 3rd Revision; WHO: Geneva, Switzerland, 2011; pp. 1-38. [CrossRef]

23. Koo, M.; Cho, A.-R.; Jeong, A.-R.; Kim, H.J.; Park, Y.-H.; Kwak, H.-S.; Hwang, I.-G. Antibiotic susceptibility and molecular typing of Enterococcus faecalis from retail pork meat products in Korea. J. Korean Soc. Appl. Biol. Chem. 2013, 56, 295-299. [CrossRef]

24. García-Solache, M.; Rice, L.B. The enterococcus: A model of adaptability to its environment. Clin. Microbiol. Rev. 2019, 32, 1-28. [CrossRef] [PubMed]

25. National Institute of Food and Drug Safety Evaluation (NIFDS). National Antimicrobial Resistance Surveillance on the Domestic and Imported Meat and Fishery Products; National Institute of Food and Drug Safety Evaluation: Chungcheongbuk-do, Korea, 2019; ISBN 1115430610. 
26. Liu, C.; Yoon, E.J.; Kim, D.K.; Shin, J.H.; Shin, J.H.; Shin, K.S.; Kim, Y.A.; Uh, Y.; Kim, H.S.; Kim, Y.R.; et al. Antimicrobial resistance in South Korea: A report from the Korean global antimicrobial resistance surveillance system (Kor-GLASS) for 2017. J. Infect. Chemother. 2019, 25, 845-859. [CrossRef] [PubMed]

27. Kwon, K.H.; Hwang, S.Y.; Moon, B.Y.; Park, Y.K.; Shin, S.; Hwang, C.Y.; Park, Y.H. Occurrence of antimicrobial resistance and virulence genes, and distribution of enterococcal clonal complex 17 from animals and human beings in Korea. J. Vet. Diagnostic Investig. 2012, 24, 924-931. [CrossRef] [PubMed]

28. Kim, M.C.; Cha, M.H.; Ryu, J.G.; Woo, G.J. Characterization of Vancomycin-Resistant Enterococcus faecalis and Enterococcus faecium Isolated from Fresh Produces and Human Fecal Samples. Foodborne Pathog. Dis. 2017, 14, 195-201. [CrossRef] [PubMed]

29. Kim, H.J.; Oh, T.; Baek, S.Y. Multidrug resistance, biofilm formation, and virulence of Escherichia coli isolates from commercial meat and vegetable products. Foodborne Pathog. Dis. 2018, 15, 782-789. [CrossRef]

30. Yoon, Y.K.; Park, G.C.; An, H.; Chun, B.C.; Sohn, J.W.; Kim, M.J. Trends of antibiotic consumption in Korea according to national reimbursement data (2008-2012) a population-based epidemiologic study. Medicine 2015, 94, e2100. [CrossRef]

31. Werner, G.; Fleige, C.; Neumann, B.; Bender, J.K.; Layer, F.; Klare, I. Evaluation of DiversiLab ${ }^{\circledR}$, MLST and PFGE typing for discriminating clinical Enterococcus faecium isolates. J. Microbiol. Methods 2015, 118, 81-84. [CrossRef]

32. Lüth, S.; Kleta, S.; Al Dahouk, S. Whole genome sequencing as a typing tool for foodborne pathogens like Listeria monocytogenes-The way towards global harmonisation and data exchange. Trends Food Sci. Technol. 2018, 73, 67-75. [CrossRef]

33. Kim, H.J.; Koo, M.; Hwang, D.; Choi, J.H.; Kim, S.M.; Oh, S.-W. Contamination patterns and molecular typing of Bacillus cereus in fresh-cut vegetable salad processing. Appl. Biol. Chem. 2016, 59. [CrossRef]

34. Cheng, G.; Ning, J.; Ahmed, S.; Huang, J.; Ullah, R.; An, B.; Hao, H.; Dai, M.; Huang, L.; Wang, X.; et al. Selection and dissemination of antimicrobial resistance in Agri-food production. Antimicrob. Resist. Infect. Control 2019, 8, 1-13. [CrossRef]

(C) 2020 by the authors. Licensee MDPI, Basel, Switzerland. This article is an open access article distributed under the terms and conditions of the Creative Commons Attribution (CC BY) license (http://creativecommons.org/licenses/by/4.0/). 